\title{
Direct Observation of Twisted Surface Skyrmions in Bulk Crystals
}

\author{
S. L. Zhang, ${ }^{1}$ G. van der Laan, ${ }^{2}$ W. W. Wang, ${ }^{3}$ A. A. Haghighirad, ${ }^{1}$ and T. Hesjedal ${ }^{1}$ \\ ${ }^{1}$ Department of Physics, Clarendon Laboratory, University of Oxford, Oxford OX1 3PU, United Kingdom* \\ ${ }^{2}$ Magnetic Spectroscopy Group, Diamond Light Source, Didcot OX11 ODE, United Kingdom \\ ${ }^{3}$ Faculty of Science, Ningbo University, Ningbo 315211, China
}

(Dated: April 17, 2018)

\begin{abstract}
Magnetic skyrmions in noncentrosymmetric helimagnets with $D_{n}$ symmetry are Bloch-type magnetization swirls with a helicity angle of $\pm 90^{\circ}$. At the surface of helimagnetic thin films below a critical thickness, a twisted skyrmion state with arbitrary helicity angle has been proposed, however, its direct experimental observation has remained elusive. Here, we show that circularly polarized resonant elastic x-ray scattering is able to unambiguously measure the helicity angle of surface skyrmions, providing direct experimental evidence that a twisted skyrmion surface state also exists in bulk systems. The exact surface helicity angles of twisted skyrmions for both left- and righthanded chiral bulk $\mathrm{Cu}_{2} \mathrm{OSeO}_{3}$, in the single as well as in the multidomain skyrmion lattice state, are determined, revealing their detailed internal structure. Our findings suggest that a skyrmion surface reconstruction is a universal phenomenon, stemming from the breaking of translational symmetry at the interface.
\end{abstract}

Magnetic skyrmions are localized, axially symmetric solutions of the nonlinear field model [1]. They are usually treated as a two-dimensional objects, which manifest themselves as magnetization swirls with non-zero topological winding number [2]. Their structures possess a nontrivial topology, leading to novel magneto-electrical transport effects [3-7] and new spin dynamic properties [8-10], enabling spintronic device concepts [11-13]. However, recent studies highlight that the surface of a three-dimensional skyrmion structure plays a crucial role and governs the physical properties of the system [14-24]. The surface is also key for device applications [16, 20-23]. Therefore, the characterization of the magnetic structure of skyrmions near the surface, which is distinctly different from its bulk character, is a challenging, yet important task with profound consequences for skyrmion science.

A skyrmion can be represented using the local magnetization unit vector $\mathbf{m}(\rho, \psi)=(\sin \Theta \cos \Phi, \sin \Theta \sin \Phi$, $\lambda \cos \Theta)$, where $(\rho, \psi)$ are the real-space polar coordinates $[1,2]$. Such an axial-symmetric expression can be understood by looking at the two structural parts. First, $\Theta=\Theta(\rho)$ describes the radial profile, i.e., the out-ofplane magnetization component $m_{z}$ that evolves from the center to the boundary of a skyrmion. $\lambda= \pm 1$ is the polarity. We define $\lambda=+1$ corresponding to the core magnetization being parallel to $+z$, and vice versa. Second, $\Phi=\psi+\chi$ is the in-plane $\left(m_{x}-m_{y}\right)$ configuration, where $\chi$ is the helicity angle [2] [see Fig. 1(d)]. The helicity is defined as $\mathcal{H}=\operatorname{sgn}(\chi)$, and the chirality is defined as $\mathcal{C}=\lambda \mathcal{H}$. For details regarding the conventions used, see supplemental materials [25]. In systems with broken inversion symmetry, the key ingredient that stabilizes magnetic skyrmions is the Dzyaloshinskii-Moriya interaction (DMI) [26], which is composed of Lifshitz in-

* Corresponding author: Shilei.Zhang@physics.ox.ac.uk variants [27]. The exact form of these invariants depends on the crystallographic symmetry, resulting in skyrmion textures with different point group symmetry and helicity angle [27]. In noncentrosymmetric bulk helimagnets with $D_{n}$ symmetry such as $\mathrm{MnSi}[28], \mathrm{Fe}_{1-x} \mathrm{Co}_{x} \mathrm{Si}$ [29], FeGe [30], and $\mathrm{Cu}_{2} \mathrm{OSeO}_{3}$ [31], the skyrmion helicity angle $\chi$ takes the values of $\pm 90^{\circ}$, commonly referred to as left- and right-handed Bloch-type skyrmions [32]. In monolayer or multilayered ferromagnetic materials $[11,12,33-35]$, skyrmions have $C_{n v}$ symmetry, which leads to $\chi=0^{\circ}$ or $180^{\circ}$, commonly referred to as divergent and convergent Néel-type skyrmions, respectively. In Heusler compounds such as MnPtSn, $D_{2 d}$ skyrmions with $\chi= \pm \pi / 2$ and negative winding number have been observed [36]. Moreover, skyrmions with $C_{n}$ and $S_{4}$ symmetry have been predicted to exist $[27,32]$.

Recent theoretical work by Rybakov et al. [14] predicted a modification to the skyrmion structure that occurs at the surface of a thin helimagnet, termed a chiral surface twist $[14,15,17-19]$. On the surface of a noncentrosymmetric bulk ferromagnet, due to the breaking of the translational symmetry, the one-dimensional chiral twist becomes evident in the saturated state. It manifests itself as a canting of the surface magnetization that deviates from the saturation magnetization direction, as, e.g., observed in $\mathrm{MnSi} / \mathrm{Si}$ epitaxial thin films [15]. Such a mechanism is also active in the case of skyrmions at the surface, leading to a deviation from the $\pm 90^{\circ}$ helicity angle for $D_{n}$-type structures due to the boundary confinement effect $[14,17]$. The formation of chiral skyrmion twists with $\chi(z) \propto z$ was studied in nanolayers of cubic helimagnets with thicknesses below the critical thickness of $8.17 L_{D}$, where $L_{D}$ is the helical modulation pitch $[14,17,18]$. This mechanism can also be regarded as the key to stabilizing the skyrmion phase in a broader range of temperatures and fields for thin films [17], extending the bulk skyrmion phase-space 
pocket. Nevertheless, such a surface skyrmion state was only indirectly observed by comparing phase diagrams of specimen with varying thickness obtained from Lorentz transmission electron microscopy (LTEM) studies [17]. Since LTEM is not sensitive to the helicity angle at the very surface of a 3D sample $[19,29,37,38]$, the direct experimental observation of twisted surface skyrmions remained elusive.
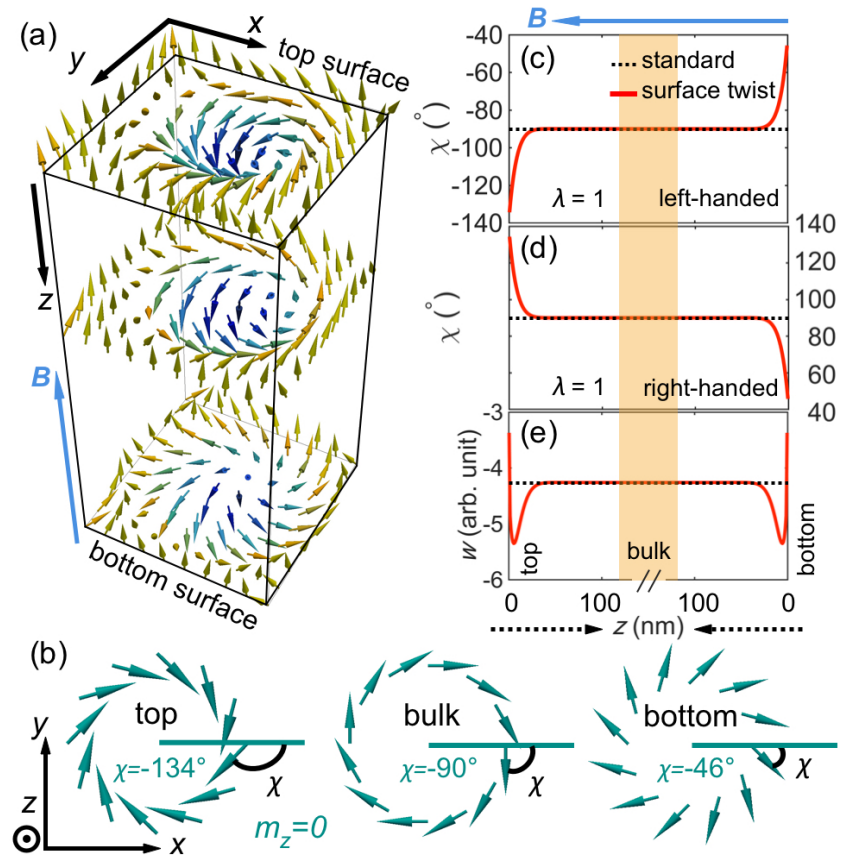

FIG. 1. (a) Illustration of the modified surface skyrmion structures in a bulk sample. The skyrmions are left-handed. We define $z=0$ as the top surface, which is probed by REXS. (b) $\chi$ is defined as the angle between the $x$-axis (red dotted line) and the $m_{z}=0$ magnetization vector (cyan) in the $x-y$ plane. (c,d) Helicity angle profile as a function of $z$ for the surface twisted state with minimized energy (red curve) and the uniformly stacked Bloch-type skyrmion structure (dotted black line), for left- (c) and right-handed (d) skyrmion system. (e) Energy density distribution, $w$, along $z$ for the twisted surface state with the $\chi(z)$ profile shown in (c) and (d) (red curve), and for the standard uniform skyrmion model (dotted line).

It is believed that $\chi= \pm 90^{\circ}$ skyrmions stack uniformly along the skyrmion tubes [17, 28]. Nevertheless, in analogy to the thin-film limit, the lack of exchange neighbors at the open boundary of a bulk helimagnet will alter the local energy density configuration [11,33,39], subsequently modifying the surface magnetic structure, i.e., a 'magnetic surface reconstruction'. The core local energy density can be written as [2, 40]

$$
w=A(\nabla \mathbf{m})^{2}+D \mathbf{m} \cdot(\nabla \times \mathbf{m})-\mathbf{B} \cdot \mathbf{m},
$$

where the first term represents the isotropic exchange interaction with exchange stiffness constant $A$, the second term is the DMI with reduced scalar DMI constant $D$ (in a continuum model), and the third term is the Zeeman interaction with applied field $\mathbf{B}$. We show that the ansatz

$$
\chi(z)=\chi_{0} e^{-z / L_{p}}-\mathcal{C} \cdot 90^{\circ},
$$

is indeed lowering the energy of the system compared to the uniformly stacked 3D skyrmion crystal (i.e., $\chi(z)=$ $\pm 90^{\circ}$ ). Figure 1(a) illustrates the magnetic surface states on the top and bottom surface of an extended bulk crystal. The applied magnetic field is perpendicular to the parallel surfaces. The helicity angle is twisted at the both surfaces, whereby the modified skyrmion structure is converging on the top surface and diverging on the bottom surface. The quantitative evolution of the helicity angle along the $z$-direction, obtained by minimizing the energy using the micromagnetic material parameters of $\mathrm{Cu}_{2} \mathrm{OSeO}_{3}$ [10], is shown in Fig. 1(c). Instead of forming the modulated $\chi(z)$ profile as predicted for thin layers [17], the twisted skyrmions undergo an exponential decay of the deformation into the bulk, eventually recovering the $\chi=-90^{\circ}$ Bloch-type skyrmion state. The helicity angle profile can thus be described by two parameters: the top surface helicity angle $\chi(0)=\chi_{0}+90^{\circ}$ and the penetration length $L_{p}$. Figure 1(d) shows the $\chi(z)$ profile for same material system with opposite chirality (i.e., $D \rightarrow-D$ ), representing the same surface twist behavior. For both cases, the energy density distribution $w$ along $z$ for the twisted state (red curve) is shown in Fig. 1(e). Compared to the uniformly stacked Blochtype skyrmion state (black line), the overall energy is lowered, suggesting that the reconstructed surface state with $\chi(0)=-134.3^{\circ}$ and $L_{p}=7.1 \mathrm{~nm}$ is more stable.

So far, the direct experimental determination of the skyrmion helicity angle remained elusive [25]. Here, we introduce a powerful experimental method for the unambiguous determination of $\chi$ of a skyrmion-carrying material using circular dichroism (CD) in resonant elastic x-ray scattering (REXS). REXS can be visualized as an elastic two-step process, in which a photon is emitted from the intermediate state after photon absorption. At the soft x-ray $\mathrm{Cu} L_{2,3}$ edge, the process is dominated by electric-dipole transitions [41, 42]. The CDREXS signal, as measured by the intensity difference of a magnetic peak using incident left- and right-circularly polarized $\mathrm{x}$ rays, can be directly related to all three components of the magnetization vector [42-45]

$$
I_{\mathrm{CD}}(\mathbf{q})=2\left|F_{1}\right|^{2} \operatorname{Im}\left\{\left[\mathbf{k}_{s} \cdot \mathbf{M}^{*}(\mathbf{q})\right]\left(\mathbf{k}_{s} \times \mathbf{k}_{i}\right) \cdot \mathbf{M}(\mathbf{q})\right\},
$$

where $F_{1}$ relates to the photon energy dependent absorption, $\mathbf{k}_{i}$ and $\mathbf{k}_{s}$ are the incidence and scattered $\mathbf{x}-$ ray wavevectors, $\mathbf{q}$ is the momentum transfer, relating to the reciprocal-space vector in the magnetic lattice, and $\mathbf{M}(\mathbf{q})$ are the Fourier components at q. A twodimensional skyrmion lattice leads to six magnetic peaks, described by the azimuthal angle $\Psi$, separated by $60^{\circ}$ 
(see $\chi=-90^{\circ}$ pattern in Fig. 2). Introducing a $2 \mathrm{D}$ skyrmion lattice structure to Eq. (3) using a computer simulation leads to a dichroic pattern in reciprocal space. As illustrated in the middle-circle gallery of Fig. 2, the six skyrmion peaks have varying circular dichroism amplitudes, and can be separated into two opposite halves with positive and negative dichroism. The direction of the line dividing the two halves (yellow and blue color on the left and right, respectively) is marked by a red arrow. At this line the dichroism vanishes, so we will call this the extinction direction. Importantly, as can be verified from Fig. 2, the angle of the extinction direction directly corresponds to the helicity angle. In other words, while $\chi$ evolves from $0^{\circ}$ to $360^{\circ}$, the extinction direction rotates by the same angle (see Supplemental Movies 1 and $2[25])$.

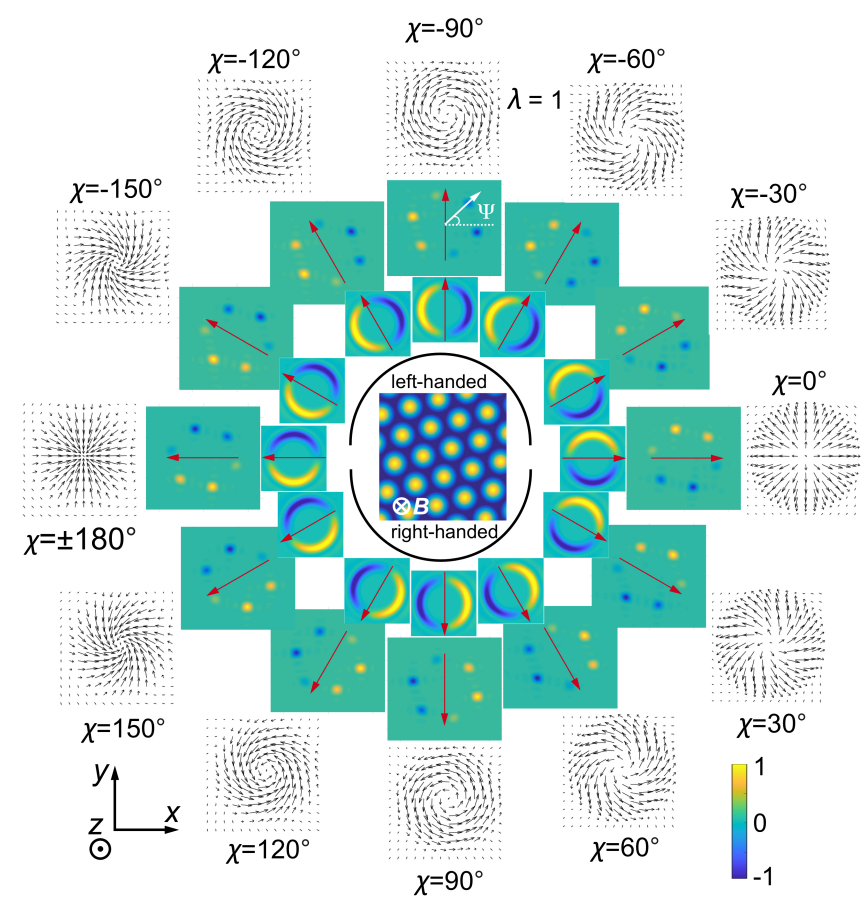

FIG. 2. Lookup 'clock' for determining the skyrmion helicity angle $\chi$. For a given magnetic field direction (here B $\|-z$ ), the extinction direction (red arrow) that separates the positive and negative dichroic peaks uniquely corresponds to $\chi$. The center image shows an arbitrary in-plane-oriented skyrmion lattice. Its motif structure carries one of the fixed $\chi$ patterns shown in the outer-circle gallery. The middle-circle gallery shows the corresponding dichroism patterns. For (inplane) disordered skyrmion lattices, the discrete spots degenerate into a dichroic ring, however, preserving the extinction direction (inner-circle gallery) which is the hallmark of the spin motif and independent of the skyrmion lattice orientation. If the magnetic field is reversed, the dichroic patterns show the inverted contrast.

Note that the angle of the extinction direction only depends on $\chi$, and is independent of other parameters. For example, for fixed $\chi$, if the skyrmion lattice is rotated azimuthally (e.g., due to a sample rotation about the momentum transfer), the azimuthal angle of the sixpeak pattern will rotate accordingly, however, the angle between the extinction direction and the $x$-axis (i.e., the intersection of $x-z$ scattering and $x-y$ surface planes) will remain the same. This is due to that fact that while the lattice is rotated, the magnetic structure of each individual skyrmion is invariant for azimuthal rotation (see Supplemental Movie 3 [25]). If one constructs a skyrmion system composed of differently (in-plane) oriented lattices, all leading to different peak positions, we find the peaks to degenerate into a dichroic ring (shown in the inner-circle gallery of Fig. 2, also see Supplemental Movie 3 [25]). This system represents the case of disordered skyrmions, each with the same $\chi$. This dichroic ring pattern is therefore representative of the spin motif, and can also be used to study many-skyrmion systems that lack long-range order, such as multilayered thin films $[11,12,33-35]$. It is then also clear that the extinction direction does not depend on the overall orientation of the sample in the setup. The CDREXS principle is summarized by the 'lookup clock' shown in Fig. 2, and can be analytically written as

$$
I_{\mathrm{CD}}(\Psi)=\lambda Y \sin (\Psi+\chi),
$$

where $Y=4\left|F_{1}\right|^{2} \pi^{2} k^{2} M_{S}^{2} \sin \theta$ is independent of $\Psi$, and $k=\left|\mathbf{k}_{i}\right|=\left|\mathbf{k}_{s}\right|, M_{S}$ is the saturation magnetization of the material, and $\theta$ is the diffraction angle. $\lambda$ strictly relates to the magnetic field direction, i.e., $\lambda=-\operatorname{sgn}(\mathbf{B})$ [2]. Therefore, in a CDREXS measurement, once the field direction is chosen, $\lambda$ is determined. Thus, $\chi$ is unambiguously identified.

We performed CDREXS experiments on polished single crystals of $\mathrm{Cu}_{2} \mathrm{OSeO}_{3}$ with well-defined cuboidal shape, measuring $2 \times 3 \mathrm{~mm}^{2}$ in the $x-y$ plane, and $1 \mathrm{~mm}$ in thickness ( $z$-direction). We measured crystals of both crystalline chiralities. REXS is an ideal technique for probing the magnetic structure near the sample surface owing to the short attenuation length of soft x-rays [21]. The skyrmion lattice phase is reached within a rather narrow temperature range of $55-57 \mathrm{~K}$ and a moderate applied field normal to the (001) surface of $\sim 20-45 \mathrm{mT}$ $[21,41,46]$. Reciprocal space mapping in the $q_{x}-q_{y}$ plane that is normal to the magnetic field direction around $(0,0,1)$ peak was then carried out [21], from which the circular dichroism signal distribution at a photon energy of $933.5 \mathrm{eV}$ was obtained. Figure 3(a) shows the CDREXS results for the skyrmion lattice phase at $56 \mathrm{~K}$, in a field of $32 \mathrm{mT}$ along the $-z$-direction. A dichroic pattern is observed, in agreement with the theoretical calculations shown in Fig. 2. From this dichroic pattern, the extinction direction can already be roughly determined. By comparing Fig. 3(a) to the 'clock' in Fig. 2, it can be concluded that the skyrmions are left-handed. If the surface skyrmions would have been Bloch-type $\left(\chi=-90^{\circ}\right)$, as in the bulk, the extinction direction should be aligned along the $y$-axis. In order to quantitatively obtain $\chi$, a 
fitting of all six peaks to Eq. (4) has to be performed, as shown in Fig. 3(b). From this fit, $\chi=-128^{\circ}$ is obtained, as marked by the extinction direction in Fig. 3(a). Therefore, it is obvious that skyrmions on the surface of the bulk crystal are twisted and converging with $\chi=-128^{\circ}$ [see inset of Fig. 3(b)].
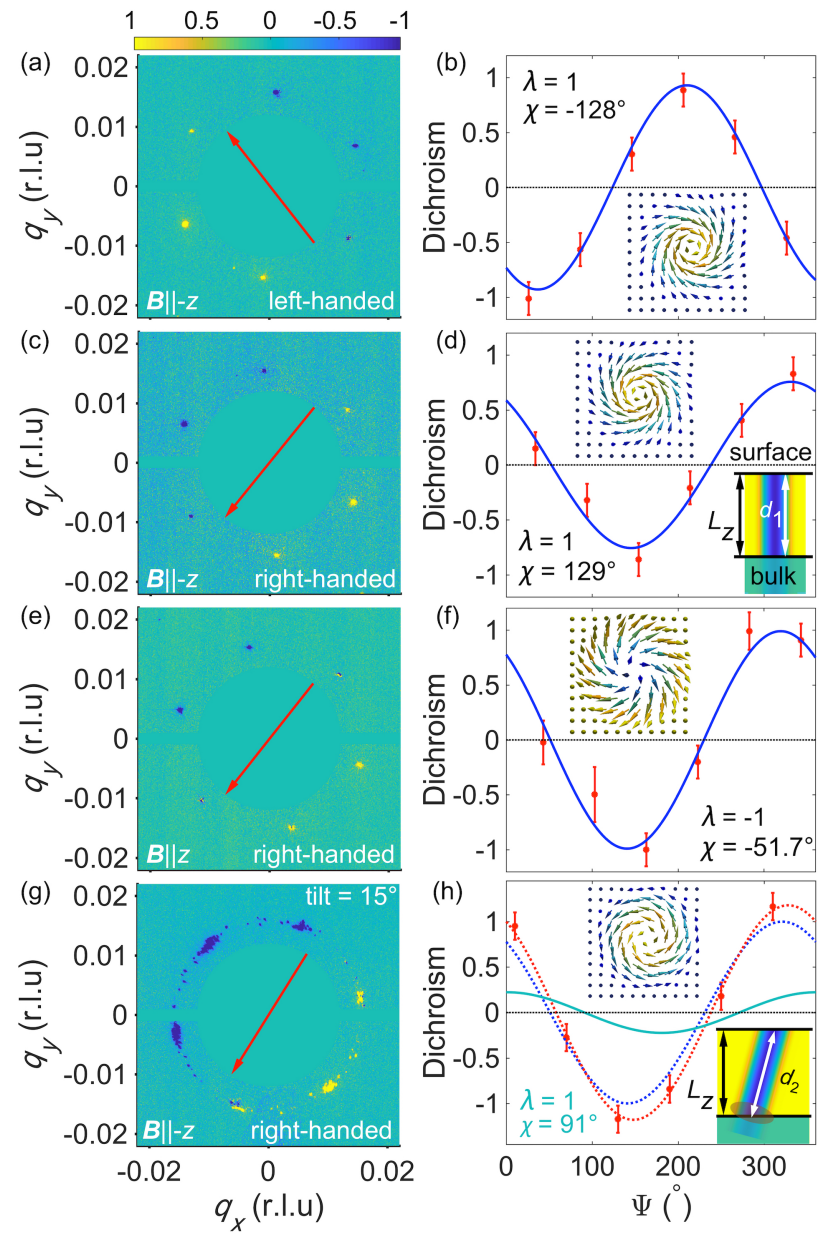

FIG. 3. Experimental determination of $\chi$ of surfacetwisted skyrmions. (a) Dichroism pattern for a left-handed $\mathrm{Cu}_{2} \mathrm{OSeO}_{3}$ crystal with $\mathbf{B} \|-z$ and the determined extinction direction (red arrow). (b) Fitting of the dichroism signal for the six skyrmion lattice peaks to Eq. (4) yields $\chi=-128^{\circ}$. The skyrmion motif is shown as the inset. (c)-(d) Dichroism pattern and fit for a right-handed crystal with $\mathbf{B} \|-z$, and (e)-(f) with reversed magnetic field direction $(\mathbf{B} \| z)$. (g) Dichroism pattern for a multidomain skyrmion lattice state, obtained by tilting the field $15^{\circ}$ off the sample normal (B $\|-z$, right-handed crystal). (h) Tilting the field also tilts the skyrmion tubes so that the x-rays probe further along the tubes $\left(d_{2}=d_{1} / \cos 15^{\circ} \approx 1.04 d_{1}\right)$. Moreover, the difference in dichroism profile $I_{\mathrm{CD}}(\Psi)$ between both measurement scenarios is due to the tube tilting (illustrated by the shaded brown area), which is $\sim 98 \mathrm{~nm}$ deep below the surface. Taking the difference, $\Delta I_{\mathrm{CD}}(\Psi)$, yields a $\chi=91^{\circ}$ profile, indicating that the bulk state is indeed a standard Bloch-type skyrmion state.

Further, a single-crystal with opposite crystalline chi- rality (as confirmed by electron backscatter diffraction [21]), but with same shape and size, was investigated. As shown in Fig. 3(c,d), by applying a $32 \mathrm{mT}$ field along the $-z$-direction, the extinction direction is opposite to the previous case. By comparing with Fig. 2, it is clear that the vortex has right-handed chirality. The extracted $\chi$ from Fig. $3(\mathrm{~d})$ is $129^{\circ}$. This suggests that although the sign of the DMI is reversed, the surface skyrmions show the same degree of twisting [see inset of Fig. 3(d)].

To investigate the magnetic state on the bottom surface of the $3 \mathrm{D}$ skyrmion crystal, we make use of the fact that the magnetic states of the bottom and top surface are exchanged when reversing the polarity of the field, as shown in Fig. 1(a). In particular, by flipping the magnetic field, the chirality is conserved, leading to both the polarity $\lambda$ and helicity $\mathcal{H}$ being reversed. In our case, when $\mathbf{B} \| z, \lambda=-1$. The experimental dichroism pattern is shown in Fig. 3(e), which appears to be identical to Fig. 3(c). However, the data has to be fitted using Eq. (4) with $\lambda=-1$. This leads to an extracted $\chi$ value of $-51.7^{\circ}$, as shown in Fig. 3(f). The divergent swirl structure [inset of Fig. 3(f)] is oppositely twisted compared with the structure on 'opposite' surface [inset of Fig. 3(d)]. These findings fully agree with the theoretical predictions for the surface twisted behavior discussed above.

Nevertheless, the measured surface twist of $\chi=129^{\circ}$ is slightly smaller than the theoretical value of $134.3^{\circ}$ [see Fig. 1(d)]. This discrepancy is possibly due to the calculated probing depth of the soft x-rays. The probing depth is mainly given by the attenuation length $L_{z}$, measuring the depth in the $z$-direction at which the incident intensity drops to $1 / e$ of its initial value [see inset of Fig. $3(\mathrm{~d})] . L_{z}$ is determined by the energy dependent absorption cross-section of the material and the angles of the incident and scattered x-rays. At the used photon energy of $933.5 \mathrm{eV}$ we calculate $L_{z} \approx 98 \mathrm{~nm}$ [47]. Although x-rays probe deep enough to 'see' the bulk state $\left(L_{z}>L_{p}\right)$, the CDREXS intensity contributions from different depths in the material are different. In general, the total intensity can be written as $I_{\mathrm{CD}}^{\mathrm{tot}}(\Psi)=\int_{0}^{\infty} I_{\mathrm{CD}}(\Psi, z) \exp \left(-z / L_{z}\right) d z$. Therefore, most of the intensity contribution comes from the top layer near the surface.

The fact that $L_{z}>L_{p}$ provides us with a strategy for retrieving the bulk skyrmion helicity angle by tilting the magnetic field. Figure 3(g) shows the dichroism pattern for a multidomain skyrmion state, obtained by tilting the field by $15^{\circ}$ away from the surface normal. The probing area is much larger than that in Fig. 3(c) due to the enlarged beam size, in order to observe the necklacelike pattern. The measurements was taken directly after CDREXS was done in the non-tilted configuration [data shown in Fig. 3(c,d)]. As discussed above [25], the extinction direction is independent of the in-plane orientation of the skyrmion domains, therefore a clear dichroic ring pattern is observed, from which a distinct extinction di- 
rection can be obtained. We further fit the dichroism peaks using Eq. (4), giving a reduced $\chi$ of $122^{\circ}$ [see Fig. $3(\mathrm{~g})]$. In order to quantitatively compare the scattering intensity to the non-tilted case, we reduced the beam size to be identical to that used in Fig. 3, and measured a single domain skyrmion lattice. The dichroism amplitude is thus normalized to the non-tilted case in Fig. 3(d), which is also plotted in Fig. 3(h) as the dotted blue curve.

The inset of Fig. 3(h) explains the reduced $\chi$ value for a tilted field. Here we make the approximation that the dichroism contribution to the total measured dichroism pattern $I_{\mathrm{CD}}^{\text {tot }}(\Psi)$ can be neglected for $z>L_{z}$. For the nontilted case, $L_{z}=d_{1}$, which is the length of the skyrmion tube probed by the x-rays (see inset of Fig. 3(d)). Therefore, $I_{1}^{\text {tot }}(\Psi)=\int_{0}^{d_{1}} I_{\mathrm{CD}}(\Psi, z) \exp \left(-z / L_{z}\right) d z$. By tilting the field, the skyrmion tubes are tilted with respect to the surface, allowing for the x-rays to effectively probe further along the tubes (depth $\left.d_{2}\right)$. The dichroism pattern can then be written as $I_{2}^{\text {tot }}(\Psi)=I_{1}^{\text {tot }}(\Psi)+\Delta I(\Psi)$, where $\Delta I(\Psi)$ will be representative for the bulk state. Figure $3(\mathrm{~h})$ shows the $\Delta I(\Psi)$ profile obtained by subtracting $I_{1}^{\text {tot }}(\Psi)$ from $I_{2}^{\text {tot }}(\Psi)$ (blue and red curves). The fitted value of $\chi=91^{\circ}$ directly suggests that bulk state skyrmions are standard right-handed Bloch-type skyrmions.

In summary, we have demonstrated a novel experimental method for the accurate measurement of the helicity angle of skyrmions. It is suitable for both ordered skyrmions, as found in noncentrosymmetric helimagnets, as well as disordered skyrmions, as found in multilayered systems. Therefore, it can be widely applied to the study of the detailed internal structure of all types of skyrmions, reaching beyond current state-ofart skyrmion characterization techniques. Moreover, we provide the first direct experimental evidence of the existence of a twisted skyrmion surface state, and show that such a surface state also exists in bulk samples. Our finding suggests that the skyrmion surface reconstruction is a universal phenomenon, owing to the breaking of translational symmetry at the interface. This varying helicity angle also highlights that $\chi$ is an additional degree of freedom of skyrmions, which may be further exploited for applications.

S.L.Z. and T.H. acknowledge financial support by EPSRC (EP/N032128/1) and W.W.W. the National Natural Science Foundation of China (Grant No. 11604169). The resonant elastic soft $\mathrm{x}$-ray scattering experiments were carried out on beamline I10 at the Diamond Light Source, UK, under proposals SI-12958, and SI-12943. We thank the I10 team for ongoing instrumental and measurement support.
[1] A. N. Bogdanov and A. Hubert, J. Magn. Magn. Mater. 138, 255 (1994).

[2] N. Nagaosa and Y. Tokura, Nat. Nanotech. 8, 899 (2013).

[3] A. Neubauer, C. Pfleiderer, B. Binz, A. Rosch, R. Ritz, P. G. Niklowitz, and P. Böni, Phys. Rev. Lett. 102, 186602 (2009).

[4] F. Jonietz, S. Mühlbauer, C. Pfleiderer, A. Neubauer, W. Münzer, A. Bauer, T. Adams, R. Georgii, P. Böni, R. A. Duine, K. Everschor, M. Garst, and A. Rosch, Science 330, 1648 (2010).

[5] X. Z. Yu, N. Kanazawa, W. Z. Zhang, T. Nagai, T. Hara, K. Kimoto, Y. Matsui, Y. Onose, and Y. Tokura, Nat. Commun. 3, 988 (2012).

[6] J. Zang, M. Mostovoy, J. H. Han, and N. Nagaosa, Phys. Rev. Lett. 107, 136804 (2011).

[7] T. Schulz, R. Ritz, A. Bauer, M. Halder, M. Wagner, C. Franz, C. Pfleiderer, K. Everschor, M. Garst, and A. Rosch, Nat. Phys. 8, 301 (2012).

[8] M. Mochizuki, Phys. Rev. Lett. 108, 017601 (2012).

[9] Y. Onose, Y. Okamura, S. Seki, S. Ishiwata, and Y. Tokura, Phys. Rev. Lett. 109, 037603 (2012).

[10] T. Schwarze, J. Waizner, M. Garst, A. Bauer, I. Stasinopoulos, H. Berger, C. Pfleiderer, and D. Grundler, Nat. Mater. 14, 478 (2015).

[11] J. Sampaio, V. Cros, S. Rohart, A. Thiaville, and A. Fert, Nat. Nanotechnol. 8, 839 (2013).

[12] W. Jiang, P. Upadhyaya, W. Zhang, G. Yu, M. B. Jungfleisch, F. Y. Fradin, J. E. Pearson, Y. Tserkovnyak, K. L. Wang, O. Heinonen, S. G. E. te Velthuis, and A. Hoffmann, Science 349, 283 (2015).

[13] R. Wiesendanger, Nat. Rev. Mater. 1, 16044 (2016).

[14] F. N. Rybakov, A. B. Borisov, and A. N. Bogdanov, Phys. Rev. B 87, 094424 (2013).

[15] S. A. Meynell, M. N. Wilson, H. Fritzsche, A. N. Bogdanov, and T. L. Monchesky, Phys. Rev. B 90, 014406 (2014).

[16] F. N. Rybakov, A. B. Borisov, S. Blügel, and N. S. Kiselev, Phys. Rev. Lett. 115, 117201 (2015).

[17] A. O. Leonov, Y. Togawa, T. L. Monchesky, A. N. Bogdanov, J. Kishine, Y. Kousaka, M. Miyagawa, T. Koyama, J. Akimitsu, T. Koyama, K. Harada, S. Mori, D. McGrouther, R. Lamb, M. Krajnak, S. McVitie, R. L. Stamps, and K. Inoue, Phys. Rev. Lett. 117, $087202(2016)$.

[18] F. N. Rybakov, A. B. Borisov, S. Blügel, and N. S. Kiselev, New J. Phys. 18, 045002 (2016).

[19] D. McGrouther, R. J. Lamb, M. Krajnak, S. McFadzean, S. McVitie, R. L. Stamps, A. O. Leonov, A. N. Bogdanov, and Y. Togawa, New J. Phys. 18, 095004 (2016).

[20] J. Müller, A. Rosch, and M. Garst, New J. Phys. 18, 065006 (2016).

[21] S. L. Zhang, A. Bauer, D. M. Burn, P. Milde, E. Neuber, L. M. Eng, H. Berger, C. Pfleiderer, G. van der Laan, and T. Hesjedal, Nano Lett. 16, 3285 (2016).

[22] C. Wang, H. Du, X. Zhao, C. Jin, M. Tian, Y. Zhang, and R. Che, Nano Lett. 17, 2921 (2017).

[23] C. Jin, Z.-A. Li, A. Kovács, J. Caron, F. Zheng, F. N. Rybakov, N. S. Kiselev, H. Du, S. Blügel, M. Tian, Y. Zhang, M. Farle, and R. E. Dunin-Borkowski, Nat. Commun. 8, 15569 (2017).

[24] K. M. Hals and K. Everschor-Sitte, Phys. Rev. Lett. 119, 
127203 (2017).

[25] "See Supplemental Material [url],".

[26] I. Dzyaloshinskii, J. Phys. Chem. Solids 4, 241 (1958).

[27] A. N. Bogdanov and D. A. Yablonskii, Sov. Phys. JETP 68, 101 (1989).

[28] S. Mühlbauer, B. Binz, F. Jonietz, C. Pfleiderer, A. Rosch, A. Neubauer, R. Georgii, and P. Böni, Science 323, 915 (2009).

[29] X. Z. Yu, Y. Onose, N. Kanazawa, J. H. Park, J. H. Han, Y. Matsui, N. Nagaosa, and Y. Tokura, Nature 465, 901 (2010).

[30] X. Z. Yu, N. Kanazawa, Y. Onose, K. Kimoto, W. Z. Zhang, S. Ishiwata, Y. Matsui, and Y. Tokura, Nat. Mater. 10, 106 (2011).

[31] S. Seki, X. Z. Yu, S. Ishiwata, and Y. Tokura, Science 336, 198 (2012)

[32] A. O. Leonov, T. I. Monchesky, N. Romming, A. Kubetzka, A. N. Boganov, and R. Wiesendanger, New J. Phys. 18, 065003 (2016).

[33] S. Heinze, K. von Bergmann, M. Menzel, J. Brede, A. Kubetzka, R. Wiesendanger, G. Bihlmayer, and S. Blügel, Nat. Phys. 7, 713 (2011).

[34] S. Woo, K. Litzius, B. Krüger, M.-Y. Im, L. Caretta, K. Richter, M. Mann, A. Krone, R. M. Reeve, M. Weigand, P. Agrawal, I. Lemesh, M.-A. Mawass, P. Fischer, M. Kläui, and G. S. D. Beach, Nat. Mater. 15, 501 (2016).

[35] O. Boulle, J. Vogel, H. Yang, S. Pizzini, D. de Souza Chaves, A. Locatelli, T. O. Mentes, A. Sala, L. D. BudaPrejbeanu, O. Klein, M. Belmeguenai, Y. Roussigné, A. Stashkevich, S. M. Chérif, L. Aballe, M. Foerster, M. Chshiev, S. Auffret, I. M. Miron, and G. Gaudin,
Nat. Nanotechnol. 11, 449 (2016).

[36] A. K. Nayak, V. Kumar, T. Ma, P. Werner, E. Pippel, R. Sahoo, F. Damay, U. K. Rößler, C. Felser, and S. S. P. Parkin, Nature 548, 561 (2017).

[37] M. J. Benitez, A. Hrabec, A. P. Mihai, T. A. Moore, G. Burnell, D. McGrouther, C. H. Marrows, and S. McVitie, Nat. Commun. 6, 8957 (2015).

[38] K. Shibata, A. Kovács, N. Kiselev, N. Kanazawa, R. Dunin-Borkowski, and Y. Tokura, Phys. Rev. Lett. 118, 087202 (2017).

[39] S. Rohart and A. Thiaville, Phys. Rev. B 88, 184422 (2013).

[40] I. E. Dzyaloshinskii, Sov. Phys. JETP 19, 960 (1964).

[41] S. L. Zhang, A. Bauer, H. Berger, C. Pfleiderer, G. van der Laan, and T. Hesjedal, Phys. Rev. B 93, 214420 (2016).

[42] G. van der Laan, C. R. Physique 9, 570 (2008).

[43] S. L. Zhang, G. van der Laan, and T. Hesjedal, Nat. Commun. 8, 14619 (2017).

[44] S. L. Zhang, G. van der Laan, and T. Hesjedal, Phys. Rev. B 96, 094401 (2017).

[45] J.-Y. Chauleau, W. Legrand, N. Reyren, D. Maccariello, S. Collin, H. Popescu, K. Bouzehouane, V. Cros, N. Jaouen, and A. Fert, Phys. Rev. Lett. 120, 037202 (2018).

[46] S. L. Zhang, A. Bauer, H. Berger, C. Pfleiderer, G. van der Laan, and T. Hesjedal, Appl. Phys. Lett. 109, 192406 (2016).

[47] G. van der Laan and A. I. Figueroa, Coord. Chem. Rev. 277-278, 95 (2014) 